\title{
The F-Analogue of Riordan Representation of Pascal Matrices via Fibonomial Coefficients
}

\author{
Naim Tuglu, ${ }^{1}$ Fatma Yesil, ${ }^{2}$ E. Gokcen Kocer, ${ }^{3}$ and Maciej Dziemiańczuk ${ }^{4}$ \\ ${ }^{1}$ Department of Mathematics, Faculty of Science, Gazi University, Teknikokullar, 06500 Ankara, Turkey \\ ${ }^{2}$ Department of Mathematics, Faculty of Art and Science, Amasya University, Ipekkoy, 05100 Amasya, Turkey \\ ${ }^{3}$ Faculty of Education, Konya Necmettin Erbakan University, Meram, 42099 Konya, Turkey \\ ${ }^{4}$ Institute of Informatics, University of Gdańsk, Wita Stwosza 57, 80-952 Gdańsk, Poland \\ Correspondence should be addressed to Naim Tuglu; tuglunaim@gmail.com
}

Received 8 February 2014; Revised 26 April 2014; Accepted 6 May 2014; Published 25 May 2014

Academic Editor: Fernando Simões

Copyright (c) 2014 Naim Tuglu et al. This is an open access article distributed under the Creative Commons Attribution License, which permits unrestricted use, distribution, and reproduction in any medium, provided the original work is properly cited.

We study an analogue of Riordan representation of Pascal matrices via Fibonomial coefficients. In particular, we establish a relationship between the Riordan array and Fibonomial coefficients, and we show that such Pascal matrices can be represented by an $F$-Riordan pair.

\section{Introduction}

Pascal matrices are infinite matrices whose entries are formed by binomial coefficients. Fibonomial coefficients are a certain class of generalized binomial coefficients, and its theory is now well understood. The Fibonomial coefficients are used to define Pascal matrices, called Pascal matrices via Fibonomial coefficients.

The Riordan group is quite easily developed but unifies many themes in enumeration. In the recent literature, special attention has been given to the concept of Riordan arrays, which is a generalization of the well-known Pascal triangle. Riordan arrays are infinite lower-triangular matrices defined by the generating function of their columns. They form a group, called the Riordan group (see [1]). Some of the main results on the Riordan group and its application to combinatorial sums and identities can be found in Sprugnoli $($ see $[2,3])$.

Setting infinite dimensional Pascal matrix via Fibonomial coefficients requires very long applications and operations, whereas it was seen that these long applications and operations can be culminated more short and in a practical way through the aid of Riordan representation. Therefore, one can obtain Pascal matrix via Fibonomial coefficients through the aid of Riordan representation.

The aim of this study is to establish the Riordan representation of Pascal matrices via Fibonomial coefficients. It is confronted by a problem while obtaining Riordan representation. Using the usual operation can not be a solution for this problem. For overcoming this problem, a new binary operation is required to define. By using this operation, $F$-analogue of Riordan representation is obtained. In particular, we show that Pascal matrices via Fibonomial coefficients of the first and the second kinds can be represented with an $F$-analogue of Riordan pair.

\section{Preliminaries}

The Fibonacci sequence is the starting point of our discussion. Thus, we briefly review some basic concepts and properties of Fibonomial coefficients. The Fibonacci numbers $F_{n}$ are defined by the initial conditions $F_{0}=0, F_{1}=1$ and the recurrence

$$
F_{n}=F_{n-1}+F_{n-2}
$$


for $n \geq 2$. Let $n$ and $k$ be integers with $n \geq k \geq 0$. Then, the Fibonomial coefficients are defined by

$$
\left(\begin{array}{l}
n \\
k
\end{array}\right)_{F}=\frac{[n]_{F} !}{[k]_{F} ![n-k]_{F} !},
$$

where $[n]_{F} !=F_{n} F_{n-1} \cdots F_{1}$ and $[0]_{F} !=1$. It can be shown that Fibonomial coefficients satisfy the following recursion relation:

$$
\left(\begin{array}{l}
n \\
k
\end{array}\right)_{F}=F_{k+1}\left(\begin{array}{c}
n-1 \\
k
\end{array}\right)_{F}+F_{n-k-1}\left(\begin{array}{l}
n-1 \\
k-1
\end{array}\right)_{F}
$$

(see [4-6]). Let $0 \leq i, j \leq n-1$. The $n \times n$ Pascal matrix, $P_{n}=\left(p_{i j}\right)$, is defined by

$$
p_{i j}= \begin{cases}\left(\begin{array}{l}
i \\
j
\end{array}\right) & \text { if } i \geq j, \\
0 & \text { otherwise. }\end{cases}
$$

The Pascal matrix via Fibonomial coefficients is denoted by $\mathscr{P}(k, m)=\left(p_{i j}\right)$ and is defined by

$$
p_{i j}= \begin{cases}\left(\begin{array}{c}
k+j m-1+i-j \\
k+j m-1
\end{array}\right)_{F} & \text { if } i \geq j, \\
0 & \text { otherwise, }\end{cases}
$$

where $k, m \in \mathbb{N}$ and $\left(\begin{array}{c}k+j m-1+i-j \\ k+j m-1\end{array}\right)_{F}$ is the Fibonomial coefficient. Specially, $\mathscr{P}(1,1)=\mathscr{P}=\left(p_{i j}\right)$ is defined by

$$
p_{i j}= \begin{cases}\left(\begin{array}{l}
i \\
j
\end{array}\right)_{F} & \text { if } i \geq j, \\
0 & \text { otherwise. }\end{cases}
$$

Similarly, the generalized Pascal matrix via Fibonomial coefficients of the first kind, $U_{n}[x]=\left(U_{n}(x, i, j)\right)$, is defined by

$$
U_{n}(x, i, j)= \begin{cases}x^{i-j}\left(\begin{array}{l}
i \\
j
\end{array}\right)_{F} & \text { if } i \geq j, \\
0 & \text { otherwise }\end{cases}
$$

and the generalized Pascal matrix via Fibonomial coefficients of the second kind, $M_{n}[x]=\left(M_{n}(x, i, j)\right)$, is defined by

$$
M_{n}(x, i, j)= \begin{cases}x^{i+j-2}\left(\begin{array}{l}
i \\
j
\end{array}\right)_{F} & \text { if } i \geq j, \\
0 & \text { otherwise. }\end{cases}
$$

Moreover, the extended generalized Pascal matrix via Fibonomial coefficients, $\Phi_{n}[x, y]_{F}=\left(\varphi_{n}(x, y ; i, j)_{F}\right)$, is defined by

$$
\varphi_{n}(x, y ; i, j)_{F}= \begin{cases}x^{i-j} y^{i+j-2}\left(\begin{array}{l}
i \\
j
\end{array}\right)_{F} & \text { if } i \geq j, \\
0 & \text { otherwise. }\end{cases}
$$

See [7-11] for details. Note that all of $\mathscr{P}, U_{n}, M_{n}$, and $\Phi_{n}$ are $n \times n$ matrices.

The Riordan group is a set of infinite lower-triangular matrices each of which is defined by two generating functions, called a Riordan pair. Any infinite matrix of this group is called a Riordan array, and Riordan arrays are generalizations of Pascal's triangle. In fact, Pascal matrices via Fibonomial coefficients are Riordan arrays, and we will show that they can be represented by a Riordan pair. To this purpose we briefly review the Riordan group and we refer to $[1-3,12,13]$ for a detailed treatment of the subject.

Definition 1 (see [1]). Let $g$ and $f$ be two functions defined by

$$
\begin{aligned}
& g(x)=g_{0}+g_{1} x+g_{2} x^{2}+\cdots, \\
& f(x)=f_{1} x+f_{2} x^{2}+f_{3} x^{3}+\cdots
\end{aligned}
$$

with $g_{0} \neq 0$. Let us denote by $(g, f)$ the infinite lowertriangular matrix whose $j$ th column is formed by the coefficients of the power series

$$
g(x) f(x)^{j}, \quad j=0,1,2, \ldots
$$

The first column of this matrix is called the 0 th column. Let $\mathscr{R}$ be the set of all infinite lower-triangular matrices defined by (11), and let $(g, f)$ and $(u, v) \in \mathscr{R}$. Then $\mathscr{R}$ becomes a group under the operation

$$
(g, f) *(u, v):=(g(u \circ f), v \circ f) .
$$

In particular, $\mathscr{R}$ is called the Riordan group and any element $(g, f)$ of $\mathscr{R}$ is called a Riordan pair. The identity element of $\mathscr{R}$ is

$$
I=(1, x)
$$

And the inverse of any $(g, f)$ is

$$
(g, f)^{-1}=\left(\frac{1}{g \circ \bar{f}}, \bar{f}\right) .
$$

Here, $\bar{f}$ is the compositional inverse of $f$; that is, $f(\bar{f}(x))=$ $\bar{f}(f(x))=x$.

\section{F-Analogue of FTRA}

In this section, firstly $*_{F}$ operation is defined and then using this operation a new theorem which is called $F$-analogue of FTRA is obtained. 
The generating functions of the Fibonomial coefficients [14-18] are

$$
\begin{gathered}
\prod_{i=1}^{n}\left(1-\alpha^{(n-i)} \beta^{(i-1)} x\right)=\sum_{k \geq 0}(-1)^{k(k+1) / 2}\left(\begin{array}{l}
n \\
k
\end{array}\right)_{F} x^{k}, \\
\prod_{i=1}^{n} \frac{1}{\left(1-\alpha^{(i-1)} \beta^{(n-i)} x\right)}=\sum_{k \geq 0}\left(\begin{array}{c}
n+k-1 \\
k
\end{array}\right)_{F} x^{k},
\end{gathered}
$$

where $\alpha=(1+\sqrt{5}) / 2$ and $\beta=(1-\sqrt{5}) / 2$. For simplicity of notation, we denote these two generating functions as follows.

Definition 2. Let $n \geq 0$; then

$$
\begin{gathered}
(1-x)_{F}^{n} \stackrel{\text { def }}{:} \prod_{i=1}^{n}\left(1-\alpha^{(n-i)} \beta^{(i-1)} x\right), \\
\frac{1}{(1-x)_{F}^{n}} \stackrel{\text { def }}{=} \prod_{i=1}^{n} \frac{1}{\left(1-\alpha^{i-1} \beta^{n-i} x\right)} .
\end{gathered}
$$

Definition 3. Let $\mathscr{F}$ denote the set of elements

$$
\begin{aligned}
\frac{t x^{m}}{(1-x)_{F}^{n}} \stackrel{\text { def }}{=} t x^{m} \prod_{i=1}^{n} \frac{1}{\left(1-\alpha^{i-1} \beta^{n-i} x\right)} \\
=t \sum_{k \geq 0}\left(\begin{array}{c}
n+k-m-1 \\
n-1
\end{array}\right)_{F} x^{k}
\end{aligned}
$$

for all integers $m, n \geq 0$ and $t \in \mathbb{R}$. Let $*_{F}: \mathscr{F} \times \mathscr{F} \rightarrow \mathscr{F}$ be a binary operation defined as follows:

$$
\begin{aligned}
\frac{t x^{a}}{(1-x)_{F}^{A}} * \frac{u x^{b}}{(1-x)_{F}^{B}} & \stackrel{\text { def }}{=} \frac{t x^{a}}{\left(1-\left(\alpha^{B} x\right)\right)_{F}^{A}} \cdot \frac{u x^{b}}{\left(1-\left(\beta^{A} x\right)\right)_{F}^{B}} \\
& =\frac{t u x^{a+b}}{(1-x)_{F}^{A+B}} .
\end{aligned}
$$

Lemma 4. The pair $\left(\mathscr{F}, *_{F}\right)$ is a monoid.

Proof. (1) Closure. Indeed, for any two elements from $\mathscr{F}$ we obtain an element from $\mathscr{F}$. That is,

$$
\frac{t x^{a}}{(1-x)_{F}^{A}} *_{F} \frac{u x^{b}}{(1-x)_{F}^{B}}=\frac{t u x^{a+b}}{(1-x)_{F}^{A+B}} \in \mathscr{F} .
$$

(2) Associativity is satisfied straightforwardly.

(3) An identity element is

$$
\frac{t^{0} x^{0}}{(1-x)_{F}^{0}}
$$

Let $g$ and $f$ belong to $\mathscr{F}$ with $g(0) \neq 0$ and $f(0)=0$. The infinite lower-triangular matrix whose $j$ th column is formed by the coefficients of the power series is

$$
g(x) *_{F} f(x)^{[j]}, \quad j=0,1,2, \ldots,
$$

where $f(x)^{[j]}:=\underbrace{f(x) *_{F} f(x) *_{F} \cdots *_{F} f(x)}$. By using new $j$ times

$*_{F}$ binary operation and (21), we obtain a representation which is the analogue of the Riordan representation. We call the representation $F$-analogue of Riordan representation and denote it by $(g, f)_{F}$.

Therefore, we can write

$$
\left(\frac{t}{(1-x)_{F}^{n}}\right)_{F} *_{F}\left(\frac{u x}{(1-x)_{F}^{m}}\right)^{[j]}=\frac{t u^{j} x^{j}}{(1-x)_{F}^{n+m j}}
$$

for any $j \geq 0$.

The following theorem is analogous to the fundamental theorem of Riordan arrays.

Theorem 5 (F-analogue of FTRA). Let $g(x), f(x) \in \mathscr{F}$ with $g(0) \neq 0$ and $f(0)=0$. The F-analogue of the fundamental theorem of Riordan arrays is

$$
(g(x), f(x))_{F}\left[\begin{array}{c}
a_{0} \\
a_{1} \\
a_{2} \\
a_{3} \\
a_{4} \\
\vdots
\end{array}\right]=\left[\begin{array}{c}
b_{0} \\
b_{1} \\
b_{2} \\
b_{3} \\
b_{4} \\
\vdots
\end{array}\right],
$$

where the generating functions of the column vectors are given, respectively, by $A(x)$ and $B(x)$. Then, equation of (23) is true if and only if the following equation holds:

$$
g(x) *_{F} A(f(x))=B(x) .
$$

Proof. Let $g(x), f(x) \in \mathscr{F}$ with $g(0) \neq 0$ and $f(0)=0$. Then, it can be written as

$$
g(x)=\frac{t}{(1-x)_{F}^{n}}, \quad f(x)=\frac{u x}{(1-x)_{F}^{m}}
$$

where $m, n \geq 0$ and $t, u \in \mathbb{R}$. In this case, the matrix turns to

$$
\left[\begin{array}{ccccccc}
\mid & & & & \cdots \\
\mid & \mid & & & \\
g & g *_{F} f^{[1]} & g *_{F} f^{[2]} & & \\
\mid & \mid & \mid & \mid & \\
\mid & \mid & \mid & \mid & \\
\vdots & & & & &
\end{array}\right]\left[\begin{array}{c}
a_{0} \\
a_{1} \\
a_{2} \\
a_{3} \\
a_{4} \\
\vdots
\end{array}\right]
$$

and then 


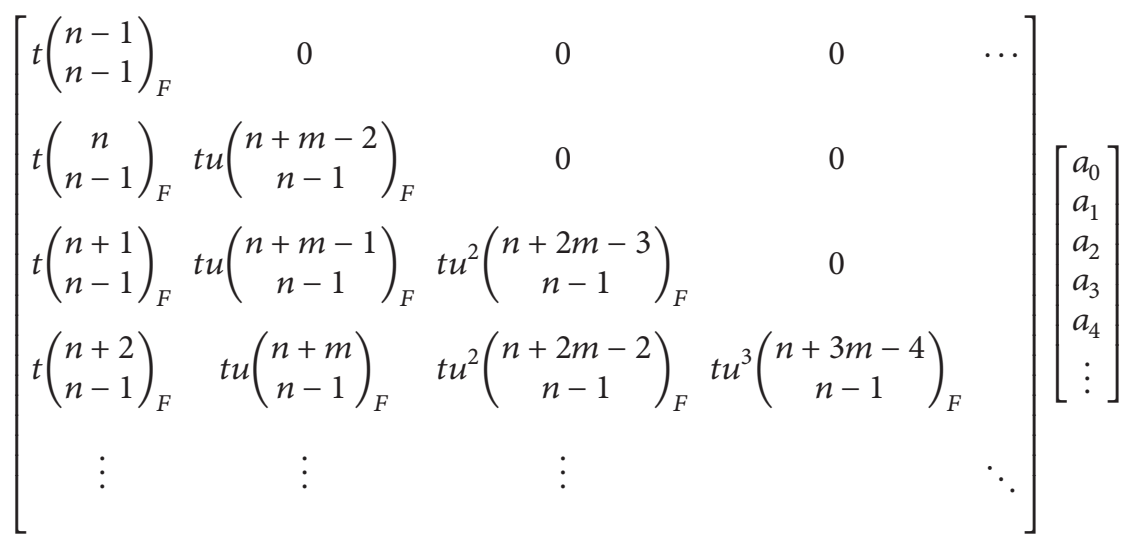

$$
\begin{aligned}
& {\left[\begin{array}{l}
a_{0} t\left(\begin{array}{l}
n-1 \\
n-1
\end{array}\right)_{F} \\
a_{0} t\left(\begin{array}{c}
n \\
n-1
\end{array}\right)_{F}+a_{1} t u\left(\begin{array}{c}
n+m-2 \\
n-1
\end{array}\right)_{F} \\
a_{0} t\left(\begin{array}{l}
n+1 \\
n-1
\end{array}\right)_{F}+a_{1} t u\left(\begin{array}{c}
n+m-1 \\
n-1
\end{array}\right)_{F}+a_{2} t u^{2}\left(\begin{array}{c}
n+2 m-3 \\
n-1
\end{array}\right)_{F} \\
a_{0} t\left(\begin{array}{l}
n+2 \\
n-1
\end{array}\right)_{F}+a_{1} t u\left(\begin{array}{c}
n+m \\
n-1
\end{array}\right)_{F}+a_{2} t u^{2}\left(\begin{array}{c}
n+2 m-2 \\
n-1
\end{array}\right)_{F}+a_{3} t u^{3}\left(\begin{array}{c}
n+3 m-4 \\
n-1
\end{array}\right)_{F} \\
\vdots
\end{array}\right]}
\end{aligned}
$$

and this yields

$$
\begin{aligned}
& a_{0} t\left(\begin{array}{l}
n-1 \\
n-1
\end{array}\right)_{F} \\
& +\left[a_{0} t\left(\begin{array}{c}
n \\
n-1
\end{array}\right)_{F}+a_{1} t u\left(\begin{array}{c}
n+m-2 \\
n-1
\end{array}\right)_{F}\right] x \\
& +\left[a_{0} t\left(\begin{array}{c}
n+1 \\
n-1
\end{array}\right)_{F}+a_{1} t u\left(\begin{array}{c}
n+m-1 \\
n-1
\end{array}\right)_{F}\right. \\
& \left.+a_{2} t u^{2}\left(\begin{array}{c}
n+2 m-3 \\
n-1
\end{array}\right)_{F}\right] x^{2}+\cdots \\
& =a_{0}\left[t\left(\begin{array}{l}
n-1 \\
n-1
\end{array}\right)_{F}+t\left(\begin{array}{c}
n \\
n-1
\end{array}\right)_{F} x\right. \\
& \left.+t\left(\begin{array}{l}
n+1 \\
n-1
\end{array}\right)_{F} x^{2}+\cdots\right] \\
& +a_{1}\left[t u\left(\begin{array}{c}
n+m-2 \\
n-1
\end{array}\right)_{F} x\right. \\
& \left.+t u\left(\begin{array}{c}
n+m-1 \\
n-1
\end{array}\right)_{F} x^{2}+\cdots\right] \\
& +a_{2}\left[t u^{2}\left(\begin{array}{c}
n+2 m-3 \\
n-1
\end{array}\right)_{F} x^{2}\right.
\end{aligned}
$$

$$
\begin{gathered}
\left.+t u^{2}\left(\begin{array}{c}
n+2 m-2 \\
n-1
\end{array}\right)_{F} x^{3}+\cdots\right]+\cdots \\
=a_{0} g(x)+a_{1}\left[g(x) *_{F} f(x)\right] \\
+a_{2}\left[g(x) *_{F} f(x)^{[2]}\right]+a_{3}\left[g(x) *_{F} f(x)^{[3]}\right]+\cdots \\
=g(x) *_{F}\left[a_{0}+a_{1} f(x)\right. \\
\left.\quad+a_{2} f(x)^{[2]}+a_{3} f(x)^{[3]}+\cdots\right] \\
=g(x) *_{F} A(f(x))=B(x)
\end{gathered}
$$

and we have our result.

\section{The F-Analogue of Riordan Representation of Pascal Matrices via Fibonomial Coefficients}

Obtaining the entries of infinite dimensional Pascal matrix via Fibonomial coefficients requires cumbersome calculations. However, there is an alternative method using the Riordan group which appears to be more convenient. To this 
purpose, we start with the following theorem in which we obtain the $F$-analogue of Riordan representation of $\mathscr{P}$.

Theorem 6. Let $\mathscr{P}(n, m)$ be the infinite Pascal matrix via Fibonomial coefficients as in (5). Then, the F-analogue of Riordan representation of $\mathscr{P}(n, m)$ is given by

$$
\mathscr{P}(n, m)=\left(\frac{t}{(1-x)_{F}^{n}}, \frac{u x}{(1-x)_{F}^{m}}\right)_{F} .
$$

Proof. We consider the infinite Pascal matrix via Fibonomial coefficients $\mathscr{P}(n, m)$. The entries of the $j$ th column are given by

$$
p_{i j}=\left[x^{i}\right] t u^{j}\left(\begin{array}{c}
n+j m-1+i-j \\
n+j m-1
\end{array}\right)_{F} \quad i=0,1,2, \ldots
$$

Let

$$
g(x)=\frac{t}{(1-x)_{F}^{n}}, \quad f(x)=\frac{u x}{(1-x)_{F}^{m}}
$$

then

$$
g(x) *_{F}(f(x))^{[j]}=\frac{t}{(1-x)_{F}^{n}} *_{F}\left(\frac{u x}{(1-x)_{F}^{m}}\right)^{[j]} .
$$

Using (22), we obtain

$$
g(x) *_{F}(f(x))^{[j]}=\frac{t u^{j} x^{j}}{(1-x)_{F}^{n+m j}} .
$$

Taking (17) into account, we have

$$
\begin{aligned}
g(x) *_{F}(f(x))^{[j]} & =\frac{t u^{j} x^{j}}{(1-x)_{F}^{n+m j}} \\
& =\sum_{i \geq 0} t u^{j}\left(\begin{array}{c}
n+j m-1+i-j \\
n+j m-1
\end{array}\right)_{F} x^{i} .
\end{aligned}
$$

This proves that the generating function of the $j$ th column of $\mathscr{P}(n, m)$ is

$$
\frac{t u^{j} x^{j}}{(1-x)_{F}^{n+m j}}
$$

In conclusion, the $F$-analogue of Riordan representation of $\mathscr{P}(n, m)$ is

$$
\left(\frac{t}{(1-x)_{F}^{n}}, \frac{u x}{(1-x)_{F}^{m}}\right)_{F}
$$

Corollary 7. Let $\mathscr{P}$ be in (6). Then, the F-analogue of Riordan representation of $\mathscr{P}$ is given by

$$
\mathscr{P}=\left(\frac{1}{(1-x)_{F}}, \frac{x}{(1-x)_{F}}\right)_{F} .
$$

Corollary 8. Let $U$ be the Pascal matrix via Fibonomial coefficients of the first kind. Then, the F-analogue of Riordan representation of $U$ is given by

$$
\begin{aligned}
& \left(\frac{1}{(1-x t)_{F}}, \frac{t}{(1-x t)_{F}}\right)_{F} \\
& =\left[\begin{array}{cccccccc}
1 & 0 & 0 & 0 & 0 & 0 & 0 & \ldots \\
x & 1 & 0 & 0 & 0 & 0 & 0 & \ldots \\
x^{2} & x & 1 & 0 & 0 & 0 & 0 & \ldots \\
x^{3} & 2 x^{2} & 2 x & 1 & 0 & 0 & 0 & \ldots \\
x^{4} & 3 x^{3} & 6 x^{2} & 3 x & 1 & 0 & 0 & \ldots \\
x^{5} & 5 x^{4} & 15 x^{3} & 15 x^{2} & 5 x & 1 & 0 & \ldots \\
x^{6} & 8 x^{5} & 40 x^{4} & 60 x^{3} & 40 x^{2} & 8 x & 1 & \ldots \\
\vdots & \vdots & \vdots & \vdots & \vdots & \vdots & \vdots & \ddots
\end{array}\right] .
\end{aligned}
$$

Corollary 9. Let $M$ be the Pascal matrix via Fibonomial coefficients of the second kind. Then, the F-analogue of Riordan representation of $M$ is given by

$$
\begin{aligned}
& \left(\frac{1}{(1-x t)_{F}}, \frac{x^{2} t}{(1-x t)_{F}}\right)_{F} \\
& =\left[\begin{array}{ccccccc}
1 & 0 & 0 & 0 & 0 & 0 & \ldots \\
x & x^{2} & 0 & 0 & 0 & 0 & \ldots \\
x^{2} & x^{3} & x^{4} & 0 & 0 & 0 & \ldots \\
x^{3} & 2 x^{4} & 2 x^{5} & x^{6} & 0 & 0 & \ldots \\
x^{4} & 3 x^{5} & 6 x^{6} & 3 x^{7} & x^{8} & 0 & \ldots \\
x^{5} & 5 x^{6} & 15 x^{7} & 15 x^{8} & 5 x^{9} & x^{10} & \ldots \\
\vdots & \vdots & \vdots & \vdots & \vdots & \vdots & \ddots
\end{array}\right] .
\end{aligned}
$$

Corollary 10. Let $\Phi$ be the extended generalized Pascal matrix via Fibonomial coefficients. Then, F-analogue of the Riordan representation of $\Phi$ is

$$
\left(\frac{1}{(1-x y t)_{F}}, \frac{y^{2} t}{(1-x y t)_{F}}\right)_{F}
$$

$$
=\left[\begin{array}{cccccc}
1 & 0 & 0 & 0 & 0 & \ldots \\
x y & y^{2} & 0 & 0 & 0 & \ldots \\
x^{2} y^{2} & x y^{3} & y^{4} & 0 & 0 & \ldots \\
x^{3} y^{3} & 2 x^{2} y^{4} & 2 x y^{5} & y^{6} & 0 & \ldots \\
x^{4} y^{4} & 3 x^{3} y^{5} & 6 x^{2} y^{6} & 3 x y^{7} & y^{8} & \ldots \\
\vdots & \vdots & \vdots & \vdots & \vdots & \ddots
\end{array}\right] .
$$

\section{Conflict of Interests}

The authors declare that there is no conflict of interests regarding the publication of this paper.

\section{References}

[1] L. W. Shapiro, S. Getu, W. J. Woan, and L. C. Woodson, "The Riordan group," Discrete Applied Mathematics, vol. 34, no. 1-3, pp. 229-239, 1991. 
[2] R. Sprugnoli, "Riordan arrays and combinatorial sums," Discrete Mathematics, vol. 132, no. 1-3, pp. 267-290, 1994.

[3] R. Sprugnoli, "Riordan arrays and the Abel-Gould identity," Discrete Mathematics, vol. 142, no. 1-3, pp. 213-233, 1995.

[4] A. K. Kwasniewski, "Fibonomial cumulative connection constants," Bulletin of the Institute of Combinatorics and Its Applications, vol. 44, pp. 81-92, 2005.

[5] E. Krot, "Further developments in finite fibonomial calculus," preprint 2004, http://arxiv.org/abs/math/0410550v2.

[6] R. Knott, “The Fibonomials," http://www.maths.surrey.ac.uk/ hosted-sites/R.Knott/Fibonacci/fib.html.

[7] G. S. Call and D. J. Velleman, "Pascal's matrices," The American Mathematical Monthly, vol. 100, no. 4, pp. 372-376, 1993.

[8] Z. Zhang, "The linear algebra of the generalized Pascal matrix," Linear Algebra and Its Applications, vol. 250, pp. 51-60, 1997.

[9] Z. Zhang and T. Wang, "Generalized Pascal matrix and recurrence sequences," Linear Algebra and Its Applications, vol. 283, no. 1-3, pp. 289-299, 1998.

[10] Z. Zhang and M. Liu, "An extension of the generalized Pascal matrix and its algebraic properties," Linear Algebra and Its Applications, vol. 271, pp. 169-177, 1998.

[11] Z. Zhang and X. Wang, "A factorization of the symmetric Pascal matrix involving the Fibonacci matrix," Discrete Applied Mathematics, vol. 155, no. 17, pp. 2371-2376, 2007.

[12] G.-y. Lee and S.-H. Cho, "The generalized Pascal matrix via the generalized Fibonacci matrix and the generalized Pell matrix," Journal of the Korean Mathematical Society, vol. 45, no. 2, pp. 479-491, 2008.

[13] P. Barry, "A study of integer sequences, Riordan arrays, Pascal-like arrays and Hankel transforms," http://repository .wit.ie/1379/.

[14] L. Carlitz, "Sequences and inversions," Duke Mathematical Journal, vol. 37, pp. 193-198, 1970.

[15] L. Carlitz, " $q$-Bernoulli and Eulerian numbers," Transactions of the American Mathematical Society, vol. 76, pp. 332-350, 1954.

[16] L. Carlitz, "Generating functions for powers of certain sequences of numbers," Duke Mathematical Journal, vol. 29, pp. 521-537, 1962.

[17] R. B. Corcino, "On p, q-binomial coefficients," Integers, vol. 8, A29, pp. 1-16, 2008.

[18] M. Dziemiańczuk, "First remark on a $\zeta$-analogue of the Stirling numbers," Integers, vol. 11, A9, pp. 1-10, 2011. 


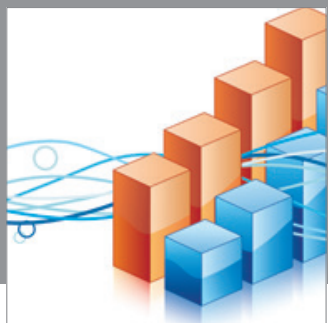

Advances in

Operations Research

mansans

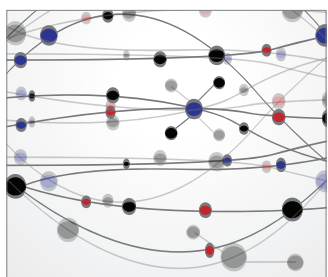

The Scientific World Journal
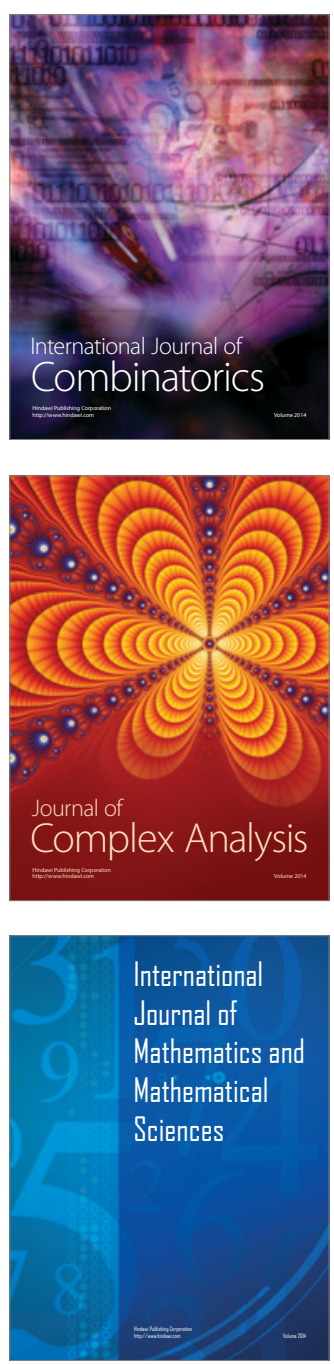
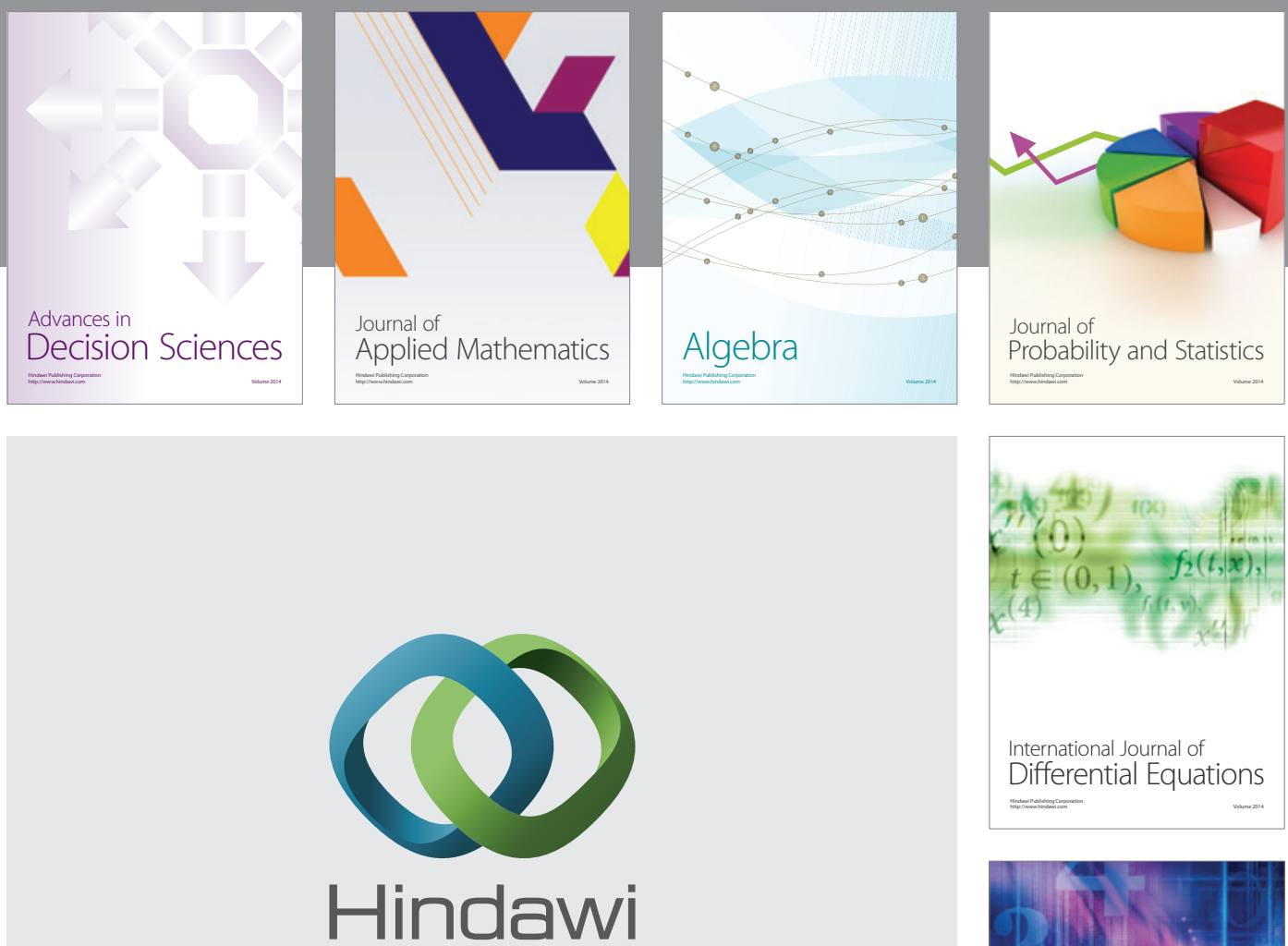

Submit your manuscripts at http://www.hindawi.com
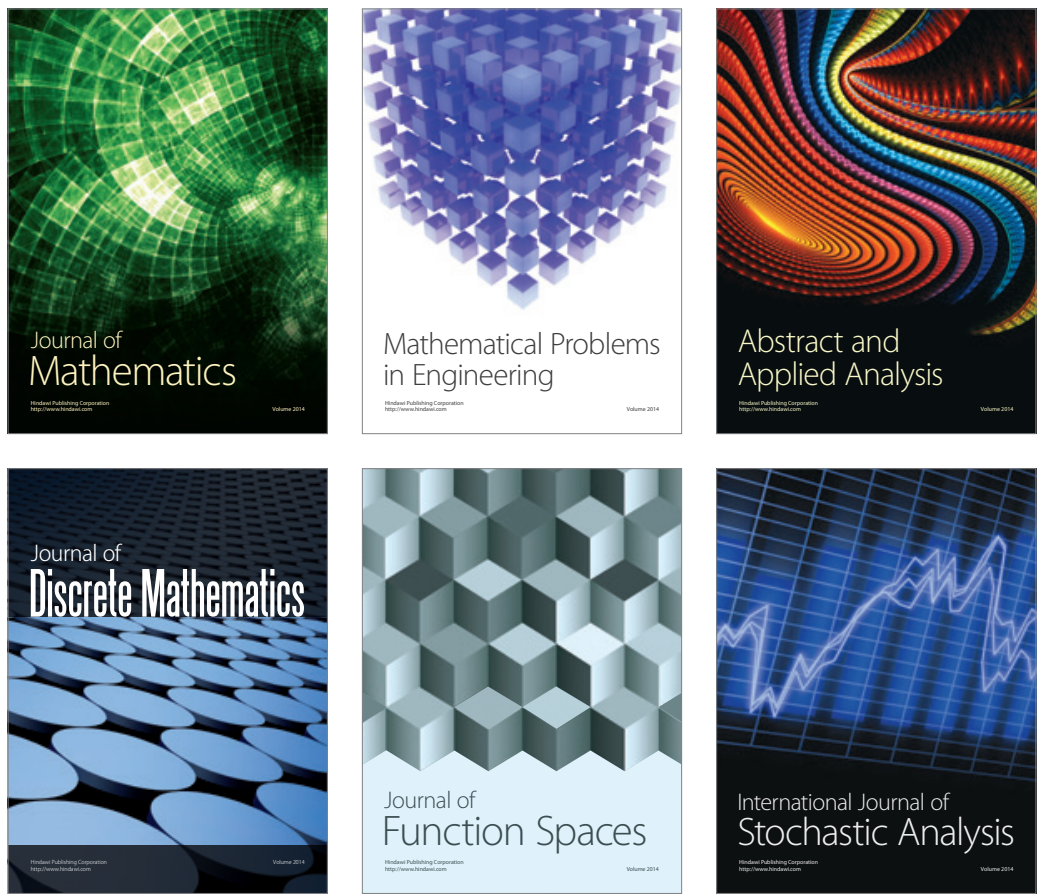

Journal of

Function Spaces

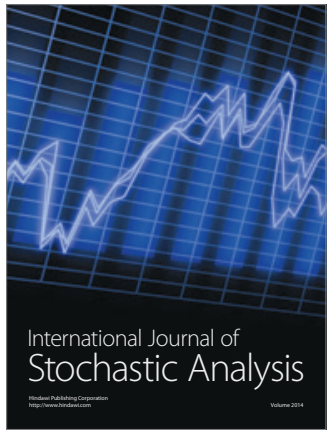

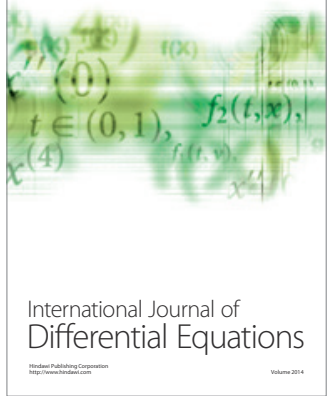
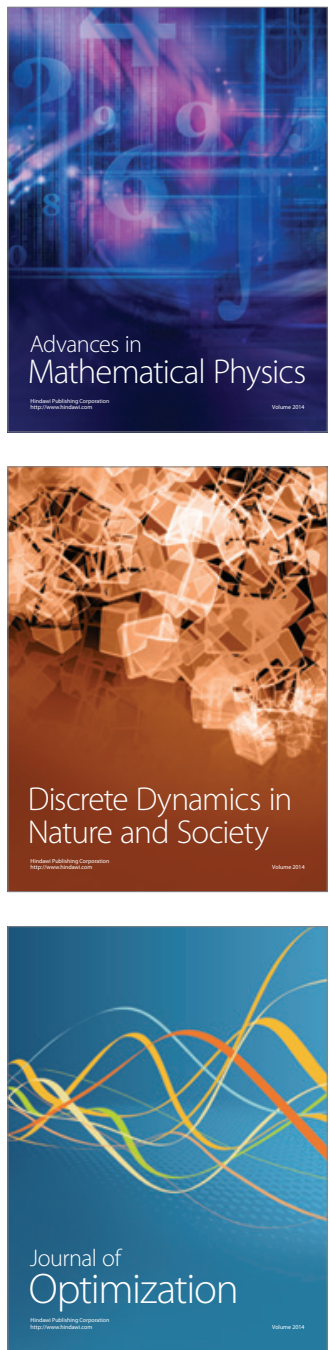